\title{
Look Beyond Cancer for Quality of Life
}

\author{
K. S. Gopinath
}

Published online: 28 February 2014

(C) Indian Association of Surgical Oncology 2014



Today patient safety and quality of life is bridging the gap for better patient care and it is a constant reminder to work systematically. There is a changing trend of utilization of molecular theranostics (Diagnostics \& Therapeutics) in the management of various cancers. The concept of personalized medicine for individual patients is increasing. We should look beyond conventional treatment and move towards personalized medicine in the management of cancer, as complications like cancer cells developing resistance to targeted drug treatment would need to be overcome. There is a need for important insights in to scientific and clinical challenges to implement national cancer genomic assessment, though it looks beyond the purview of clinical oncology.

This issue highlights some of the aspects, "look beyond cancer care" and have a good quality of life. In this issue the article by Gunasheela and Gunasheela highlighted utility of Strategies for Fertility Preservation in Young Patients with Cancer: A Comprehensive Approach. The article concludes

\footnotetext{
K. S. Gopinath $(\bowtie)$

Ambuja Health Care, \# 2/2 Patalamma Temple street, Basavanagudi, Bangalore 560004, India

e-mail: gopiijso@gmail.com
}

that there is a need for cryopreservation of oocytes and ovarian tissue for younger cancer patients for future reproductive life.

The editorial by Prof. Donald Weaver Dendritic Cell Based Immunotherapy of Cancer with Cell Penetrating Domains has enabled us to understand development of new technology of cloned full length MAGE-A3 in the expression vector in frame with CPD and successfully demonstrated that the recombinant CPD-MAGE-A3 fusion proteins efficiently entered into cytosol of DCs without affecting the phenotype of the cells. We are in the process of generating CTLs to show tumor cell killing. We believe that this method will generate efficient tumor lysing CTLs and pave way for better therapeutic cancer vaccine generation.

Ismail Jatoi et al. have analysed and trying to understand Effect of Local Therapy on Breast Cancer Mortality: Is There an Age-Interaction. They concluded by evidence for such an age-interaction is derived from comparison of the breast cancer hazard curves in women with high-risk and low-risk tumors, and analysis of the mammography screening trials. Neo-adjuvant systemic therapy may eventually prove useful in modulating the effects of local therapy.

It is a great honor to write this information, as we are stepping in to the fifth year of publication of the journal. The last 4 years has seen growth of the journal in scientific contents, administrative apparel and accreditation by various recognized bodies.

This year we are to trying to bring some change in the format with the support of editorial board I hope we will be able to do it.

However last year witnessed a breakthrough in oncology in the Indian subcontinent and a well managed Indian Cancer Congress 2013 was organized jointly by Indian Association Surgical Oncology, AROI, ISMPO, ISO with cooperation of 26 subspecialty sections catering to the needs of all concerned with cancer care. The total number of abstract presentations 
exceeded to nearly 500 in Surgical Oncology indicating the growth of the specialty of Oncology.

I appreciate excellent work and professionalism in bringing out the issue of IJSO. I always say your constructive criticism for progress of science is always welcome.

The dedicated work by Dr. K. Harish associate editor, Joint editor Dr. Chintamani and Dr.Raghu Pillara Setty in streamlining the indexing process by Govt. of India needs special mention. I have to thank the administrative staff Mr. J. Suresh (Dayananda Sagar College of Engineering), Dr. Shivananda Swamy and other staff members at Ambuja Health Care who helped in carrying out successful publication of IJSO. The efficient functioning of Springer's staff in publishing the journal is highly appreciated. 